\title{
"Les Névroses" de Maurice Rollinat: une tentative de retour/recours fin-de-siècle à la nature
}

\section{Patrick Thériault}

\section{(2) OpenEdition}

10 Journals

\section{Édition électronique}

URL : http://journals.openedition.org/studifrancesi/16086

DOI : 10.4000/studifrancesi. 16086

ISSN : 2421-5856

Éditeur

Rosenberg \& Sellier

\section{Édition imprimée}

Date de publication : 1 juillet 2019

Pagination : 42-55

ISSN : 0039-2944

\section{Référence électronique}

Patrick Thériault, «"Les Névroses" de Maurice Rollinat: une tentative de retour/recours fin-de-siècle à la nature », Studi Francesi [En ligne], 187 (LXIII | I) | 2019, mis en ligne le 01 avril 2020, consulté le 24 janvier 2021. URL : http://journals.openedition.org/studifrancesi/16086 ; DOI : https://doi.org/ 10.4000/studifrancesi.16086

\section{(c) (i) $\odot$}

Studi Francesi è distribuita con Licenza Creative Commons Attribuzione - Non commerciale - Non opere derivate 4.0 Internazionale. 


\title{
"Les Névroses" de Maurice Rollinat: une tentative de retour/recours fin-de-siècle à la nature
}

\begin{abstract}
Maurice Rollinat's Les Névroses (1883) exhibits a distinctively original feature as a work of poetry; it draws on two kinds of inspiration that are usually regarded as categorically opposed if not categorially exclusive, that, artificialist, of decadent poetry, on the one hand, and that, naturist, of rustic poetry, on the other. A close reading of the work suggests that the relationships between these two poetic trends are to be conceived not only in terms of opposition, as critics would normally do, but also in terms of hybridization. We intend here to draw attention to the main aspects of this singular hybridization of aesthetic codes and values, and to capture its global symbolic motivation by presenting it as a tentative decadent return/resort to nature. Through this aesthetic gesture, as we will suggest, Rollinat extends the "quivering" of modern sensitivity to natural order. By doing so, he allows for the use of pastoral and animal motifs which are usually excluded from poetic representation of fin-de-siecle modernity. Conversely, he also provides a new possibility of revitalisation or regeneration for the Baudelairian poetic paradigm. We will postulate that the fact that this possibility remains in the end unexploited by other poets and that Rollinat came to be associated, both in the eyes of his contemporaries and in the memory of posterity, with a rather unfortunate epigone of Baudelaire could be attributed to two reasons: his poetic ethos generally maladapted to decadent aesthetics and, more importantly, the radicalism of his endeavour which brings the decadent logic of the sensation to a point of self-contradiction pragmatically untenable.
\end{abstract}

C'est lui, le naturel dans l'étrange, si on peut dire de l'étrange qu'il soit naturel...

Jules Barbey d'Aurevilly ${ }^{1}$

À Paris où il est «monté» depuis son Berry natal, Maurice Rollinat s'illustre dans les années 1870 et au début des années 1880 en conjuguant une poésie d'inspiration baudelairienne et des talents de compositeur interprète. En s'accompagnant au piano, il en vient à donner voix à une figure particulièrement tourmentée de poète maudit, qui lui vaut d'être l'homme du jour de la bohême montmartroise et, au-delà, de certains salons mondains. En 1883, la parution de ce qui s'imposera comme son œuvre maîtresse, Les Névroses², consacre ce personnage spleenétique et sensationnel, hyperboliquement étrange, en même temps qu'elle annonce sa sortie de scène: de fait, quelques mois plus tard, supportant mal le désamour d'une partie de la critique à son

(1) Un poète à l’horizon!, «Le Constitutionnel», $1^{\text {er }}$ juin 1882. Cet article est reproduit dans le dossier «Les Névroses et la presse (1882-1883)» fourni en appendice (pp. 383-432) de l'édition des Névroses composant le deuxième tome des Euvres de Rollinat (éd. R. Miannay, Paris, Minard, 1972, «Lettres modernes/Bibliothèque introuvable»). La présente citation se trouve à la page 388 de cet ouvrage.

(2) Ph. Martin-Lau en a récemment proposé une réédition (Paris, Éditions du Sandre, 2012, 422 pp.). C'est à cette édition que nous renvoyons, en indiquant, pour tout passage auquel nous faisons référence, le folio entre parenthèses dans le corps du texte. 
endroit, Rollinat quitte la vie parisienne et retourne dans la Creuse, où il continuera à écrire et passera l'essentiel de son temps jusqu'à sa mort en $1903^{3}$.

L'architecture interne des Névroses translate sur le plan esthétique l'opposition ville/campagne autour de laquelle s'articule la trajectoire biographique et institutionnelle de Rollinat: quatre sections marquées par des thèmes décadents y encadrent une autre, intitulée «Les refuges», à dominante pastorale. En première approximation, l'œuvre semble être le fait d'un poète double ou bifrons, qui d'un côté prolonge l'esthétique de la modernité de Poe et Baudelaire et, de l'autre, trouve «refuge» dans les plaisirs immémoriaux de la contemplation bucolique. Tout en étant représentative du décadentisme - et de son parti pris artificialiste, que Des Esseintes résumera lapidairement, l'année suivante, en constatant que «la nature a fait son temps!» ${ }^{4}-$, elle semble préfigurer le retour à la terre et le provincialisme poétique qui s'affirmeront au début des années 1890 en réaction à ce même décadentisme $e^{5}$. Cette juxtaposition d'inspirations hétérogènes suffirait à inscrire le recueil de Rollinat au compte des bizarreries esthétiques auxquelles la modernité poétique, animée par son esprit de «chercherie» ${ }^{6}$ caractéristique, a donné lieu. Mais cette appréciation générale et contrastante est quelque peu simplificatrice ${ }^{7}$; elle a le défaut de masquer ce qu'on peut considérer comme le foyer de la véritable originalité du recueil et, plus largement peut-être, de l'ensemble de la production poétique de Rollinat. La lecture attentive des Névroses invite en effet à relativiser l'opposition entre poésie rustique et poésie décadente et à considérer également leurs rapports en termes d'immixtion ou d'hybridation. Elle permet de constater que l'antique muse des champs y noue des liens étroits, parfois même indissociables, avec la «muse malade» de Baudelaire ${ }^{8}$. Pour peu qu'on y réfléchisse, le motif du refuge qui est au cœur de la structure d'ensemble du recueil suggère d'ailleurs moins une dichotomie qu'une dialectique, donc une certaine interdépendance, entre ses matières. Mais c'est dans le cadre d'un certain nombre de poèmes, situés à l'intérieur comme à l'extérieur de la section «Les refuges», que s'opère véritablement et dans toute sa singularité cette hybridation de codes et de valeurs esthétiques en laquelle nous proposons ici de reconnaître une

(3) Pour toute considération d'ordre biographique, cfr. R. Miannay, Maurice Rollinat, poète et musicien du fantastique, Paris, Minard, 1981, «Lettres modernes/Bibliothèque introuvable». Dans une perspective bibliographique, on consultera avec profit le recensement des œuvres de Rollinat disponibles dans internet que propose R. CROSNIER sur le site Maurice Rollinat sur internet. URL: http://www.crcrosnier.fr/rollinat/ rollinat-internet.htm (site consulté le 13 février 2019).

(4) J.-K. Huysmans, À Rebours, éd. M. Fumaroli, Paris, Gallimard, 1977, «Folio Classique», p. 103.

(5) M. Décaudin, La Crise des valeurs symbolistes. Vingt ans de poésie française (1895-1914), Toulouse, Privat, 1969, «Universitas», pp. 33-37. Cfr. aussi M. Gorenc, Les Poètes du pays natal (1870-1890). L'exemple de Jean Aicard et de François Fabié, Paris, Champion, 2014, «Babeliana», pp. 7-61.

(6) Ch. Baudelaire, Révélation magnétique (1848), in Baudelaire journaliste. Articles et chroniques, éd. Alain Vaillant, Paris, Flammarion, 2011, «GF», p. 82.

(7) Et elle induit d'autres interprétations semblablement schématisantes, comme en témoigne, dans un registre affectif, ce jugement d'un contemporain du poète: «Il y a en Rollinat deux hommes: et c'est dans cette duplicité même du consolant et du désespérant que se déchire son âme en proie au doute inquiet. Les deux faces de son talent sont distinctes, on pourrait dire contradictoires: Janus n'a pas deux têtes sans que l'une tourne le dos à l'autre» (P. Nogent, Maurice Rollinat, «L'Opinion», 28 février 1883, cité in RollinAT, Euvres, t. II cit., p. 416).

(8) Ces liens sont d'ailleurs bien antérieurs aux années 1880. Précisons que la composition des Névroses est pour une bonne part contemporaine de celle du premier recueil, à dominante pastorale, de Rollinat, Dans les brandes, paru (quelque peu tardivement) en 1877. C'est dire que, sur le plan de son évolution de créateur, il est aussi impossible d'instaurer une dichotomie nette entre les inspirations rustique et décadente de Rollinat (cfr. C. Le Guillou, Correspondance et création poétique chez Maurice Rollinat, in La Lettre et l'ouvre. Perspectives épistolaires sur la création littéraire et picturale au XIX siècle, éd. P. AuraixJonchière, C. Croisille et É. Francalanza, Clermont-Ferrand, Presses universitaires Blaise-Pascal, 2009, «Écritures de l'intime», p. 111). 
forme de retour/recours fin-de-siècle à la nature. Le rapprochement que nous désignons par cette expression n'a ni l'étendue ni le degré de réflexivité qui en feraient une poétique dûment constituée, mais il relève d'une tendance qui s'avère suffisamment forte, croyons-nous, pour dénoter une tentative poétique. Une tentative qui présente l'intérêt critique de ne pas avoir de précédent ou de parallèle facilement reconnaissable ${ }^{9}$, tout en s'avérant être, par son caractère inachevé et aberrant, «monstrueusement» exemplaire du décadentisme et de ses mariages contre-nature. De fait, l'un des rares motifs définitoires que l'on puisse attribuer avec quelque certitude au décadentisme est bien de «tente[r] d'unifier en un tout cohérent des éléments qui ressortissent à des ordres dissemblables» ${ }^{10}$.

À travers sa tentative d'hybridation, Rollinat semble d'abord soucieux d'hystériser la (représentation de la) nature, de communiquer le «frisson» de la sensibilité moderne à l'ordre naturel, c'est-à-dire à cela même que Poe et Baudelaire «ne virent pas $\gg^{11}$. Ce faisant, sous le signe paradoxal d'une angoisse envahissante, il confère une nouvelle vie et veine littéraires à des motifs pastoraux et animaliers qu'on aurait d'emblée cru incompatibles avec les paramètres esthétiques de la modernité. Inversement - à une époque particulièrement mouvementée de l'histoire littéraire où, comme on sait, le baudelairisme ambiant requiert des jeunes poètes désireux de nouveauté et de reconnaissance qu'ils négocient ingénieusement, par une forme d'appropriation qui soit à la fois fidèle et créative, l'héritage esthétique des Fleurs du mal -, il indique aussi une possibilité de revitalisation ou de renouvellement du paradigme poétique. Que cette possibilité soit en fin de compte restée à peu près inexploitée et que Rollinat ait été assimilé, aux yeux de la plupart de ses contemporains et dans la mémoire de la postérité, à un épigone infortuné de Baudelaire ou, moins défavorablement, au plus célèbre des «petits poètes» baudelairiens ${ }^{12}$, et ce, en dépit d'un talent artistique qu'on ne peut réduire à ses dispositions de comédien et de musicien -, nous en verrons la raison dans son ethos poétique globalement mal accordé à l'esprit finde-siècle, mais surtout dans la radicalité de sa tentative, qui a pour effet de pousser la logique de la sensation sous-jacente au décadentisme à un point d'auto-contradiction pragmatiquement intenable.

(9) En modernité, les cas de poète ayant prisé les poisons de la vie décadente ou simplement la vie de bohème avant d'entendre ou de réentendre l'appel de la nature ne sont pas inusités - pensons à Léon Cladel ou Charles Frémine, qui furent les amis de Rollinat. De même, il n'est pas rare d'y rencontrer des auteurs se servant de décors rustiques pour mettre en scène des narrations d'inspiration décadente - pensons à Paul Adam et à son conte La Glèbe. En revanche, à notre connaissance, aucun écrivain n'y tente une immixtion des paradigmes décadent et rustique aussi fondamentale que celle - de profondeur catégorielle - de Rollinat dans Les Névroses.

(10) D. Grojnowski, Aux commencements du rire moderne: l'esprit fumiste, Paris, José Corti, 1997, p. 58. Nous employons les vocables «fin-de-siècle», «baudelairisme» et «modernité» («poétique») dans un sens équivalent à celui de «décadentisme», donc pour exprimer, de manière sommaire mais selon nous tout à fait légitime et opératoire, l'imaginaire baudelairien tel qu'il se déploie historiquement à partir de la publication des Fleurs du mal et en vient à s'exprimer dans la production poétique des années 1860, 1870 et 1880 à travers les penchants bien connus de l'époque pour la négativité, le pessimisme, la provocation, le raffinement artificialiste et l'exploitation de thèmes morbides.

(11) L. BLOY, Les artistes mystérieux: Maurice Rollinat, «Le Foyer illustré», 17 septembre, 24 septembre et $1^{\text {er }}$ octobre 1882, article reproduit in Rollinat, Euvres, t. II. cit., p. 393.

(12) G. Peylet, Les Évasions manquées ou les illusions de l'artifice dans la littérature «fin de siècle», Genève-Paris, Slatkine, 1986, p. 213. Son influence sur ses contemporains semble difficile à établir, du fait qu'aucun historien du symbolisme ne l'a véritablement établi, comme s'en étonne G. Michaud (Message poétique du symbolisme, Paris, Nizet, 1961, p. 244, note infrapaginale n. 51). On ne peut conclure pour autant qu'elle fut «nulle», comme le fait L. MARquèze-Pouey (Le Mouvement décadent en France, Paris, P.U.F., 1986, «Littératures modernes», p. 101). 


\section{Une nature névrosée}

La névrotisation de la nature à laquelle procède Rollinat s'indique d'abord par un signe très manifeste, presque obsédant: le frisson. Depuis 1859 et la célèbre lettre de Hugo sur le «frisson nouveau» ${ }^{13}$ induit par les Fleurs du mal, ce motif s'est imposé comme l'un des mots-emblèmes du baudelairisme, de son ethos et de sa production poétiques à caractère nerveux. Mieux sans doute que toute autre toute allégorèse finde-siècle ${ }^{14}$, il dit l'esprit et l'ethos de cette poésie nouvelle vouée à creuser et nourrir la «fringale d'intensité perceptive et d'ultra-sensibilité» ${ }^{15}$ dont témoigne la modernité naissante.

Dans Les Névroses, le frisson apparaît avec une telle force d'impression (il exprime souvent une peur paranoïaque extrême) et avec une telle force d'évidence (le mot et ses dérivés reviennent plusieurs dizaines de fois dans le recueil et il sert de titre à l'une de ses pièces liminaires (38)) qu'il acquiert une intensité idiosyncratiquement rollinatienne. Mais son intérêt critique, en l'occurrence, réside dans le fait qu'il déborde les catégories du nervosisme fin-de-siècle, les attendus de l'esthétique décadente et, plus en amont, l'imaginaire fantastique et gothique du romantisme frénétique. Il ne sert pas seulement à caractériser l'effet ou l'affect nerveux de la poésie nouvelle (la peur, qui est le «frisson noir de la pensée» (39), le «frisson clandestin» (325), l'insolite (290) ou bien la fureur (275)) ni l'être hystérique du véritable poète (paradigmatiquement Poe, «grand Ténébreux qu'on lit en frissonnant!» (83, 40, 321)). Au-delà de ces emplois admis, le frisson s'associe chez Rollinat à pratiquement tout: les individus (au premier chef les femmes $(39,54,136,305)$, c'est un fait entendu), les végétaux $(192,221)$ et les animaux de toutes sortes (sauterelles (164), vipères (201), pouliches (175)...), les astres $(195,320)$, les phénomènes naturels (147, 166), le temps (262), la mort (40), les produits de l'industrie humaine... Bref, c'est l'ensemble des étants, ici, qui est transi de frissons et comme soumis à une même tonalité fondamentale - une même «Stimmung», dirait le philosophe - s'exprimant dans le registre de la frilosité (149), du frétillement (164), du tressaillement (168), de la vibration $(190,262)$, du tremblotement $(156,237)$, du frémissement (366), du reniflement $(158) \ldots$

En parcourant transversalement toutes les catégories d'être, le frisson module et modèle ainsi la représentation d'une vie naturellement malade, congénitalement névrotique. Il porte la névrose de l'individu moderne, et de cet individu par excellence névrotique et moderne qu'est le poète Rollinat ${ }^{16}$, à l'échelle du cosmos. En résulte une image de la nature pour le moins insolite qui, tout en reconduisant le subjectivisme romantique et en épousant les contours hypertrophiés du Moi créateur, est imprégnée d'une négativité et d'une morbidité toutes décadentes ${ }^{17}$. Cette nature peut-elle encore servir

(13) Lettre de Victor Hugo à Charles Baudelaire, 6 octobre 1859, reproduite in A. GuYAux, Charles Baudelaire. Un demi-siècle de lectures des "Fleurs du mal" (1855-1905), Paris, P.U.P.S., 2007, «Mémoire de la critique», p. 297. Cfr. la préface de cet ouvrage, pp. 67-72.

(14) Sur l'allégorèse en contexte décadent, cfr. C. Coquio, La «Baudelairité» décadente: un modèle spectral, in «Romantisme», 1993 , n. 82, p. 96.

(15) H. Lemaître, La Poésie depuis Baudelaire, Paris, Armand Colin, 1965, «U/Lettres françaises», p. 28.

(16) Ch. Bruneau le qualifie ainsi de «Décadent typique» (cité par MARQuÈze-Pouey, Le Mouvement décadent en France cit., p. 101, note infrapaginale n. 33). C'est également le jugement qui ressort de la représentation - caricaturale et malveillante, certes, mais significative - qu'en donne F. CHAMPSAUR dans son roman à clef Dinah Samuel sous les traits du personnage Michel Maury, alias Michel Spleen (Paris, Ollendorff, 1889, pp. 119-121)

(17) Le long poème «La ruine» (333-337), dans l'avant-dernière section du recueil, en offre une illustration exemplaire; tout en participant au regain d'intérêt de l'époque pour les décors gothiques, il compose ce qu'on pourrait appeler un paysage d'âme... névrotique. Sur les rapports entre décadentisme et gothique, 
de refuge au poète? La question se pose avec d'autant plus d'insistance que le thème du refuge, on l'a dit, revêt une importance déterminante dans le recueil: il en est le cœur matériel et symbolique. Or, comme ce thème capte à plus vaste échelle toute une partie de l'imaginaire culturel du XIX ${ }^{e}$ siècle ${ }^{18}$, le type d'appropriation qu'en fait Rollinat permet de mesurer, sur le plan critique, le degré d'originalité de sa proposition esthétique.

S'il est évident, à cet égard, qu'il s'inscrit en faux par rapport à ses contemporains décadents, qui se détournent de la nature pour s'évader dans une forme ou une autre d'intériorité domestique ou psychique (érudition, souvenirs, paradis artificiels...), sa représentation valorisante de l'univers rural ne se contente pas de répéter, à contretemps, le locus amœenus de l'imaginaire (néo)classique ou le point de fuite hors civilisation du romantisme. Régis Miannay note que le refuge naturel chez Rollinat n'offre très souvent qu'un abri relatif, à la fois précaire et temporaire ${ }^{19}$. Même dans un poème au titre aussi peu ambigu que «Fuyons Paris», il est seulement l'affaire d'un séjour de «quelques semaines» ${ }^{20}$ et les possibilités de ressourcement ou de dédommagement symbolique qu'y trouve le poète s'avèrent limitées, insuffisantes qu'elles sont à transformer radicalement son être. Plus encore, ce refuge apparaît hanté par la ville, si bien qu'en l'évoquant, Rollinat parle «du citadin beaucoup plus que du campagnard $\gg^{21}$. C'est un expédient, dont on peut conjecturer qu'il marque, au-delà de l'impossibilité pour l'homme Rollinat de revenir en arrière, à un style de vie et de création jugé plus purement rustique, la difficulté pour la modernité d'un retour aux codes et à la symbolique traditionnels de l'idylle ${ }^{22}$.

Un poème comme «Le petit lièvre», situé dans la section «Les refuges», semble donner raison à cette interprétation. En première lecture, cette pièce d'inspiration naïve (mais d'une polymétrie rare et quelque peu artiste, banvillienne) semble figurer l'un de ces asiles de fraîcheur, de ces «sursauts de vie saine dans la présence de la Nature» ${ }^{23}$ qu'évoque habituellement la critique pour désigner les poèmes champêtres des Névroses, en les opposant à l'ensemble massif des autres poèmes relevant clairement de la frénésie et de la fébrilité décadentes. Mais, en s'y penchant avec un peu plus d'attention, on découvre qu'à travers son thème animalier «Le petit lièvre» allégorise une figure de poète pour qui la campagne est loin de constituer un refuge hermétiquement séparé de la ville et de ses préoccupations symboliques:

Brusque, avec un frisson

De frayeur et de fièvre,

On voit le petit lièvre

S'échapper du buisson.

Ni mouche ni pinson;

Ni pâtre avec sa chèvre,

cfr. J. PRungnaud, Gothique et décadence. Recherche sur la continuité d'un mythe et d'un genre au XIXe siècle en Grande-Bretagne et en France, Paris, Honoré Champion, 1997, «Littérature générale et comparée», pp. 241-376.

(18) S. Jouve, Obsessions et perversions dans la littérature et les demeures à la fin du dix-neuvième siècle, Paris, Hermann, 1996, «Savoir/Lettres», p. 110.

(19) Maurice Rollinat, poète et musicien du fantastique cit., pp. 174-175.

(20) Il s'agit du poème liminaire de Dans les brandes. M. Rolinat, CEuvres, t. I (Dans les brandes), éd.

R. Miannay, Paris, Minard, 1971, «Lettres françaises/Bibliothèque introuvable», pp. 75-79.

(21) Ibid., p. 75.

(22) C'est ce que suggère l'évolution du genre de l'idylle au XIXe siècle, qui est l'objet à la fois de profondes transformations et de multiples réinvestissements. Cfr. V. BONEU, L'Idylle en France au XIX' siècle, Paris, Presses de l'Université Paris-Sorbonne, 2014, «Lettres françaises».

(23) H. Lapaire, Rollinat. Poète et musicien, Paris, Librairie Mellottée, 1930, «Le génie des provinces françaises», p. 118. 
La chanson

Sur la lèvre.

Tremblant au moindre accroc,

La barbe hérissée

Et l'oreille dressée,

Le timide levraut

Part et se risque au trot,

Car l'aube nuancée

N'est pas trop

Avancée. [...]

Terrains mous, terrains durs,

En tout lieu son pied trotte;

Et poudreux, plein de crotte,

Ce rôdeur des blés murs

Hante les trous obscurs

Où la source chevrote,

Les vieux murs

Et la grotte. [...]

L'if qui se rabougrit,

Le roc vêtu d'ouate

Où le genêt s'emboîte,

La forêt qui maigrit,

La mare qui tarit,

L'ornière creuse et moite:

Tout sourit

Et miroite.

Et dans le champ vermeil

Où s'épuise la sève,

Le lièvre blotti rêve

D'un laurier sans pareil;

Et toujours en éveil

Il renifle sans trêve

Au soleil

Qui se lève. (156-158)

Rollinat profite de ces quelques vers, au rythme vif et fébrile, à l'image du lièvre, et de cette mise en scène champêtre, où «[t] out sourit|Et miroite», pour s'adonner à un petit jeu réflexif: il esquisse son autoportrait de poète. Le lièvre, en tant que motif d'identification, peut d'abord étonner: il ne fait pas partie des animaux qu'on retrouve traditionnellement dans les totems d'écrivain. Mais il s'avère en vérité tout à fait congruent avec la persona que Rollinat donne à lire par ailleurs dans son recueil, en particulier dans ses poèmes à dominante décadente. De fait, cette créature fragile et fiévreuse, tout en tremblements et en frissons, animée par une frayeur constante, affiche certaines marques caractéristiques de la malédiction littéraire: elle a la «barbe hérissée»; elle est crottée (et rappelle en cela le stéréotype du «poète crotté» $\left.{ }^{24}\right)$; sa manière d'être générale - c'est un «rôdeur» - dénote un ethos assez

(24) Sur le stéréotype du «poète crotté», cfr. Cl. CRIsTIN, Aux origines de l'bistoire littéraire, Grenoble, Presses universitaires de Grenoble, 1973, «Hypothèses», pp. 13-28. 
clairement baudelairien; et les «trous obscurs» qu'elle fréquente sont analogues aux abymes de l'intériorité psychique et aux gouffres de la négativité sociale qui font le principal sujet de l'esthétique moderne. Mais, bien plus qu'un jeu de travestissement animalier, l'évocation de ce petit lièvre embraye vers la situation symbolique et institutionnelle du poète; elle comporte des indices paratopiques, comme dirait Dominique Maingueneau, révélateurs ${ }^{25}$. On remarque à cet effet que, si ce petit lièvre est «toujours en éveil», à l'affût, c'est non seulement en raison de sa nature nerveuse mais parce qu'il est animé par le rêve d'un «laurier sans pareil», c'est-à-dire - l'allusion ne fait guère mystère, cette fois - d'une reconnaissance authentique, singularisante. Plus encore, cet animal de l'aube obéit et préside à une dynamique de l'entre-deux, qui correspond, structurellement, à celle de toute paratopie ${ }^{26}$ : il évolue au seuil d'une lumière qui est à la fois source de danger et de tentation et qui, comme telle, semble renvoyer à la publicité de l'espace littéraire, cette publicité que Maurice Rollinat, suivant une ambivalence qui est l'un des traits les plus constants de sa personnalité, et plus sûrement encore de sa stratégie d'auteur, ne cessera de craindre et de rechercher. C'est ainsi que ce poème naturiste parle à mots plus ou moins couverts de la ville en tant que foyer de consécration symbolique - de ce Paris capitale de la République des lettres avec lequel Rollinat ne coupera jamais définitivement les ponts ${ }^{27}$ - et qu'il révèle un refuge naturel plus clairsemé, plus exposé à l'extériorité sociale et urbaine qu'on le croirait d'entrée de jeu. Un refuge décidément plus buissonnier que forestier, pourrait-on dire, à l'image des brandes chères à l'auteur ${ }^{28}$.

\section{2. Être naturellement décadent}

Comme le suggère cet exemple, l'hybridation rollinatienne des esthétiques rustique et décadente apparaît habituellement sur fond de nature, comme présence frissonnante, et perturbatrice, de l'esprit fin-de-siècle à l'intérieur de schèmes naturalistes traditionnels. Elle prend généralement la forme de ce qu'on pourrait appeler un «romantisme nerveux» ${ }^{29}$ ou, encore, suivant une expression sans doute mieux faite

(25) Cfr. D. Maingueneau, Le Discours littéraire. Paratopie et scène d'énonciation, Paris, Armand Colin, 2004, «U/Lettres», pp. 70-116; et Trouver sa place dans le champ littéraire. Paratopie et création, Louvainla-Neuve, Academia-L'Harmattan, 2016, «Au cœur des textes», pp. 25-35.

(26) D. Maingueneau, Le Discours littéraire cit., pp. 106-109.

(27) Son biographe note à ce sujet: «[...] s'il se tint à l'écart des milieux littéraires après la publication des Névroses, il continua de s'informer sur ce qui se passait à Paris en ce domaine grâce à un petit groupe d'amis fidèles. Il fut attentif à ce qu'on écrivait ou disait sur ses livres, comme le prouvent de nombreuses pages d'En errant, qui sont, après un silence d'une vingtaine d'années, une réponse aux critiques qu'on lui avait adressées» (R. Miannay, Maurice Rollinat, poète et musicien du fantastique cit., p. 378).

(28) Notons que le paratexte du «Petit Lièvre», qui inclut, comme une très large partie des poèmes des Névroses, une dédicace à un acteur parisien du monde des lettres, en l'occurrence Léon Bloy, redouble et souligne ce que son sous-texte suggère: à savoir la prégnance des enjeux relatifs à l'économie littéraire et, par métonymie, de la ville (jusque) dans un cadre thématique pastoral. La dédicace, c'est bien connu, revêt une fonction hautement symbolique. Elle est prisée par les écrivains - de la modernité, tout particulièrement - au titre d'occasion de médiation, de don et de contre-don, dans le système d'échanges que décrit l'économie symbolique de la littérature. Elle suscite et entretient des liens institutionnels et, de manière plus générale, extériorise et contribue à produire le champ littéraire comme espace social d'inscription et de circulation des textes. Sur les implications symboliques de la dédicace, cfr. P. Durand, Mallarmé. Du sens des formes au sens des formalités, Paris, Seuil, 2008, «Liber», pp. 126-128.

(29) Ce terme, du reste, n'est pas inédit; tout comme l'expression «mysticisme des nerfs», il est employé par Hermann Bahr en 1904 pour décrire la peinture impressionniste (B. LANGENBRUCH, L'Impressionnisme et la littérature allemande. Les modalités d'un double transfert culturel, in Impressionnisme et littérature, sous la dir. de G. Gengembre, Y. Leclerc et F. Naugrette, Mont-Saint-Aignan, Presses universitaires de Rouen et du Havre, 2012, p. 112). 
pour rendre compte de la tension maximaliste des Névroses, un romantisme extranerveux. Mais il arrive au poète d'inverser la focale et de procéder à son mélange caractéristique d'inspirations en évoquant une sorte d'irruption naturiste dans un cadre urbain et baudelairien. C'est un tel retour du (refoulé) naturel que met en scène «La pluie», dans la section «Les spectres». Ce poème est d'autant plus intéressant sur le plan critique qu'il noue une relation intertextuelle très serrée avec l'œuvre de Baudelaire et, par ce biais, nous permet d'appréhender le geste esthétique de Rollinat en ses termes les plus fondamentaux: à savoir comme une tentative motivée d'abord par la revendication et le dépassement du tout ce dont Baudelaire, à l'époque, est le nom. En voici les strophes les plus signifiantes:

Lorsque la pluie, ainsi qu'un immense écheveau

Brouillant à l'infini ses longs fils d'eau glacée,

Tombe d'un ciel funèbre et noir comme un caveau

Sur Paris, la Babel hurlante et convulsée,

J'abandonne mon gîte, et sur les ponts de fer,

Sur le macadam, sur les pavés, sur l'asphalte,

Laissant mouiller mon crâne où crépite un enfer,

Je marche à pas fiévreux sans jamais faire halte.

La pluie infiltre en moi des rêves obsédants

Qui me font patauger lentement dans les boues,

Et je m'en vais, rôdeur morne, la pipe aux dents,

Sans cesse éclaboussé par des milliers de roues.

Cette pluie est pour moi le spleen de l'inconnu:

Voilà pourquoi j'ai soif de ces larmes fluettes

Qui sur Paris, le monstre au sanglot continu,

Tombent obliquement lugubres, et muettes. [...]

En ruminant tout haut des poèmes de fiel,

J'affronte sans les voir la flaque et la gouttière;

Et mêlant ma tristesse à la douleur du ciel,

Je marche dans Paris comme en un cimetière.

Et parmi la cohue impure des démons,

Dans le grand labyrinthe, au hasard et sans guide,

Je m'enfonce, et $\mathrm{j}$ 'aspire alors à pleins poumons

L'affreuse humidité de ce brouillard liquide.

Je suis tout à la pluie! À son charme assassin,

Les vers dans mon cerveau ruissellent comme une onde:

Car pour moi, le sondeur du triste et du malsain,

C'est de la poésie atroce qui m'inonde. (298-299)

On le constate, ce poème est si étroitement tissé dans le texte des Fleurs du mal qu'il constitue une forme de centon poétique en hommage à Baudelaire. Pratiquement tous les motifs qu'il convoque sont baudelairiens: la pluie comme «écheveau», Paris ville «hurlante», la posture hystérique du poète, le rôdeur, le spleen, l'atroce... Il n'est pas jusqu'à l'image sur laquelle il repose et pivote finalement - celle de l'inté- 
riorité du poète comme terre inondée - qui ne soit empruntée aux Fleurs du mal3. Toutefois, sous le couvert de la plus stricte conformité à l'imaginaire de son maître, Rollinat s'en démarque, et ce, d'une manière même fondamentale, puisqu'elle engage le rapport du poète et de la poésie à la nature. Il s'en distingue plus spécifiquement à la faveur d'un jeu intertextuel se déployant à partir d'un poème des Tableaux parisiens, «Le soleil» ${ }^{31}$. De ce texte, «La pluie» de Rollinat s'avère offrir une image en quelque sorte symétrique mais inversée, une image en miroir. Ce jeu d'inversion fait fond sur une parenté: les deux poèmes font intervenir une réalité naturelle symboliquement chargée, le soleil, la pluie, en relation avec une certaine caractérisation de la poésie $^{32}$. En revanche, le sens et l'importance qu'ils accordent respectivement à ces réalités naturelles diffèrent radicalement. Chez Rollinat, la pluie domine complètement le poète; elle ne fait pas que l'imprégner et le nourrir, elle le submerge, le noie. En s'y exposant, le poète cherche lui-même à se déposséder, à s'abandonner corps et âme à la nature. Aussi l'exclamation de la dernière strophe: «Je suis tout à la pluie!» s'entend-elle à la fois comme un cri de défaite et de triomphe. Elle signale moins la transformation du poète que son retour et son appartenance, foncière, à la nature. Chez Baudelaire, au contraire, le poète n'abdique pas face à l'élément naturel: il est en position de rivalité avec le soleil, lequel, par là même, permet de préciser analogiquement la nature ou l'antinature de la poésie, mais reste étranger à l'expérience de la création. Plus encore, loin de se livrer au soleil, le poète finit par le dominer, comme il apparaît dans la proposition comparative du $17^{\mathrm{e}}$ vers: «Quand, ainsi qu'un poète, il descend dans les villes». À l'encontre de qu'on s'attendrait en bonne tradition rhétorique, c'est le soleil, ici, qui est à l'image du poète, qui est réduit à l'état de comparé. Par extension, c'est l'ordre de la nature qui se subordonne à celui de la culture, instancié par l'écriture.

Si donc, dans son poème, Rollinat s'oppose au scénario victorieux de Baudelaire et met en scène son abdication de poète face à la pluie, son absorption dans le giron de la nature, il n'en travaille pas moins - sur le plan des enjeux symboliques où reconduit ici, de manière expresse, le jeu intertextuel - à son affirmation auctoriale. En effet, toute son ode à la pluie, et en particulier la scène de dépossession jouissive où elle culmine («Je suis tout à la pluie!»), semble avoir pour but de résumer en termes pathétiques ce qui fait sa singularité de poète en regard de Baudelaire et du baudelairisme ou, plus simplement, la nouveauté spécifique de sa modernité: à savoir l'étiologie naturelle de sa névrose. Ses épousailles ou retrouvailles avec la pluie sont l'occasion privilégiée d'affermir son statut d'écrivain en faisant béer les fondements

(30) Dans le sonnet «L'ennemi» (Ch. Baudelaire, Euvres complètes, t. I, éd. Cl. Pichois, Paris, Gallimard, 1975, «Bibliothèque de la Pléiade», p. 16).

(31) Ibid., p. 83.

(32) On peut préciser la parenté de ces poèmes en notant qu'ils ont en commun d'assimiler la création poétique à une expérience initiatique, épreuve impliquant de la part du poète un départ (l'«abandon» de son gîte), un isolement du groupe social (une avancée «sans guide» «dans le grand labyrinthe» de la ville) et la participation, à l'issue d'un parcours à la fois réel et imaginaire, à une forme d'«affrontement» à caractère épique engageant le monde physique, le langage et la subjectivité. Cette épreuve est la condition d'accès et la voix d'ascèse de la production, ou de la culture, poétique: qui s'y livre peut espérer trouver, «le terreau ameubli» de son intériorité créatrice, les «vers» désirés - suivant le calembour auquel recourent tous deux, après tant d'autres, Rollinat et Baudelaire. Dans l'évocation de cette expérience, l'élément naturel prend la valeur d'une source de vie et de mort: le soleil baudelairien est «nourricier» et «cruel»; la pluie rollinatienne abreuve l'âme du poète, suscite ses facultés créatrices, tout en induisant chez lui un état «atroce». Que le premier revête une symbolique paternelle et la seconde une dimension maternelle et matricielle, il n'importe: la nature, dans les deux cas, cumule des attributs similairement opposés (et similairement conformes au caractère bivalent de nombreuses figures archétypales). Sur la symbolique solaire chez Baudelaire, cfr. M. EIGELDINGER, La symbolique solaire dans la poésie de Baudelaire, «Revue d'histoire littéraire de la France» 2, 67, avril-juin 1967, pp. 357-374. 
naturels de sa posture auctoriale, en exhibant les racines profondes du mal, la névrose, qui définit sa poésie et l'«être même» de son personnage littéraire. Tout semble se disposer ici pour fixer l'image sur laquelle se fonde la prétention distinctive de Rollinat, image de marque qui en fait un «homme d'une nervosité naturelle» ${ }^{33}$. L'expression est de Barbey d'Aurevilly, qui fut avec Léon Bloy et, plus tôt, George Sand, comme on sait, l'appui le plus précieux du poète dans sa lutte à la reconnaissance institutionnelle. Il semble aussi avoir été l'interprète le plus lucide de sa démarche symbolique. Dans les deux longs articles en forme de plaidoyer qu'il a consacrés aux Névroses ${ }^{34}$, dont le premier paraît avant leur publication, le romancier semble avoir discerné avec beaucoup de justesse l'avantage différentiel que Rollinat cherche à cultiver à travers l'ensemble de son discours et de son jeu social. Avantage dont il faut souligner le caractère différentiel ou distinctif, puisqu'il suppose que Rollinat serait naturellement névrosé à la différence de Poe et de Baudelaire, dont la névrose aurait besoin d'être induite ou du moins stimulée par une forme d'altération étrangère, l'alcool et la drogue:

On le sait maintenant, Edgar Poe lampait en enfilée douze verres d'eau-de-vie, avant d'écrire; Baudelaire se jetait à l'opium et à la morphine, et ils sont morts tous les deux pour avoir voulu raviver à ce prix les défaillances de leur génie! et c'est par là que Rollinat, tout en leur ressemblant, diffère d'Edgar Poe, l'ivrogne sublime, et de Baudelaire, l'homme au hatchiss des Paradis artificiels.

Pour être poétiquement diabolique, Rollinat, cet homme d'une nervosité naturelle n'a besoin ni de piments, ni de moxas, ni de cantharides. Il n'a ni habileté, ni subtilité, ni rétorsion, ni préméditation d'art, scélérate... ${ }^{35}$

Pour Barbey, c'est donc en tant que décadent naturellement intoxiqué que l'auteur des Névroses mérite de trouver place, et bonne place, dans la filiation de la modernité. La plupart des critiques sympathiques à Rollinat endosseront la croyance à cette «nature spéciale» ${ }^{36}$, qui récupère à son profit, en achevant de la biologiser, l'immémoriale mystique de l'élection et de la malédiction poétiques. Ce levier symbolique ne permet pas simplement au poète de «compenser son infériorité artistique par rapport à ses illustres devanciers ${ }^{37}$; il semble aussi conçu pour l'exhausser au-dessus d'eux. La stratégie auctoriale de Rollinat est plus audacieuse qu'il n'y paraît: faisant fond sur la doxa selon laquelle la névrose est l'affect le plus caractéristique du décadentisme, elle consiste à laisser entendre que le poète «à la nervosité naturelle» est plus radicalement, et par là plus autbentiquement, décadent que ses prédécesseurs ${ }^{38} \ldots$

Sans exclure, évidemment, qu'il ait été l'objet de certaines prédispositions à la maladie mentale, il importe ainsi de souligner comment Rollinat, sinon construit, du moins exploite sa névrose à des fins de distinction et de reconnaissance symboliques.

(33) J. Barbey D’Aurevilly, Un poète à l'horizon!, «Le Constitutionnel», $1^{\text {er }}$ juin 1882, cité in Rollinat, Euvres, t. I, cit., p. 387.

(34) Ibid., pp. 384-388 et «Les Névroses» par M. Maurice Rollinat (chez Charpentier), «Le Pays», 6 juillet 1883, cité in ibid., pp. 426-432.

(35) Ibid., p. 387.

(36) H. LAPAIRE, Rollinat. Poète et musicien cit., p. 83.

(37) R. Miannay, Maurice Rollinat, poète et musicien du fantastique cit., p. 330.

(38) Si on en juge à l'héritage littéraire qu'a laissé Rollinat, il est certain que cette stratégie n'a pas atteint son but. Mais on peut penser qu'elle n'est pas restée sans effets, dans la mesure où elle semble avoir durablement lié le nom du poète à la question de la sincérité et polarisé la réception des Névroses entre les défenseurs d'un Rollinat sincère (c'est-à-dire authentiquement névrosé) et les tenants d'un Rollinat histrionique (c'est-à-dire faussement névrosé). Le responsable de la récente édition des Névroses au Sandre, dans son introduction, reconduit d'ailleurs ces paramètres (op. cit., pp. 18-19 et 25). 
La naturalisation de la névrose est la pièce maîtresse de sa stratégie auctoriale, telle qu'elle semble tout entière guidée, et déniée, par son désir d'apparaître authentique. Dans le «champ figuratif» ${ }^{39}$ que représente l'espace littéraire, où il s'agit, comme le rappelle Daniel Oster, non seulement de faire croire, mais de «faire croire qu'on ne veut pas faire croire» ${ }^{40}$, la sincérité est davantage l'effet recherché par le discours que sa condition d'énonciation. Aussi doit-elle rester sujette à caution. Il faut d'autant plus s'en méfier que Rollinat, en prétendant l'ancrer dans un fond paysan, comme il apparaît, cherche à en faire un usage maximal. Pour être audacieuse, sa tentative, toutefois, n'est peut-être pas judicieuse: transformer la névrose en un mal primairement naturel, donc congénital et spontané, c'est lui nier sa réflexivité, c'est-à-dire cela même qui en fait une réalité éminemment «littéraire», en particulier depuis Poe et Baudelaire; c'est la dissocier de cet espace excédentaire de pensée, ce pli ou repli de conscience, en lequel le poète de la modernité jouit et souffre de son décalage par rapport au monde et par rapport à lui-même, en lequel il fait l'expérience de l'écart entre soi et les autres, la pensée et l'action, le désir et le réel. Presque tous les commentateurs de Rollinat ont relevé le caractère incongru et comme lacunaire de sa névropathie littéraire, en reconnaissant en lui un poète plus baudelairien que Baudelaire par la puissance de provocation des images, l'intensité dramatique, la tonalité pathétique, mais finalement très peu réflexif. «Il n'analyse pas» ${ }^{41}$, note peu amènement Barrès, dans un article célèbre. C'est un fait que cet imitateur parfois fidèle jusqu'à la servilité aux thèmes et aux affects nerveux des Fleurs du mal n'offre pas de véritable plongée dans les profondeurs de la psychè humaine. On aurait de la difficulté - même si, comme on l'a vu, certains de ses poèmes se doublent d'une trame métapoétique - à lui accorder cette vertu de lucidité qui, de Poe à Valéry, en passant par Baudelaire et Mallarmé, constitue l'un des marqueurs les plus caractéristiques du poète moderne. Électrisées par une tension qui leur confère souvent l'allure de «quelque furieuse pantomime» ${ }^{42}$, marquées par une force d'impression pouvant confiner à une forme d'《horrible plat» ${ }^{43}$, ses Névroses sont pour ainsi dire moins névrotiques qu'hystériques.

\section{Les antinomies de la déraison décadente}

Incidemment, le cas de Rollinat nous permet de constater ceci: esthétique du mensonge, le décadentisme place celui qui s'en réclame dans une impossibilité énonciative similaire à celle du paradoxe du menteur. Dès lors que sa névrose complique ou empêche son adhésion aux rôles sociaux, que sa conscience suraigüe l'amène à reconnaître le caractère factice (ou «fictionnel», comme dirait Mallarmé) de sa propre identité et des constructions symboliques en général, à commencer par le langage, le décadent est un personnage qui ne peut se prendre ni être pris au sérieux. On

(39) D. Oster, L'Individu littéraire, Paris, P.U.F., 1997, «Écriture», p. 4. Oster y définit l'espace figuratif comme la fiction «de personne» que construit chacune des interventions publiques de l'écrivain: ses apparitions, ses paroles, ses poses, ses fréquentations, mais aussi ses omissions, ses silences et ses évitements.

(40) ID., Rangements, Paris, P.O.L., 2001, p. 221. Sur la sincérité comme valeur littéraire, cfr. N. MANNING, Rhétorique de la sincérité. La poésie moderne en quête d'un langage vrai, Paris, Champion, 2013, «Bibliothèque de littérature générale et comparée».

(41) La sensation en littérature. La folie de Charles Baudelaire: Baudelaire, Verlaine, Mallarmé, Rollinat, des Esseintes [2 $2^{\mathrm{e}}$ partie], «Les Taches d'encre», 5 décembre 1884, p. 33.

(42) Ibid.

(43) J. Laforgue, cité par A. Guyaux, Charles Baudelaire. Un demi-siècle de lectures des "Fleurs du mal" (1855-1905) cit., p. 86. 
ne s'étonne pas que ses modèles littéraires les plus emblématiques - des Esseintes, Monsieur Phocas, Adoré Floupette - en soient aussi et indistinctement des dénonciations parodiques. On ne s'étonne pas non plus que ses plus grands représentants aient voulu déjouer les attentes sincéristes de l'idéologie littéraire ambiante et se soient gardés de se dire en toute transparence: Poe en faisant d'un certain esprit de mystification l'un des traits du génie ${ }^{44}$; Baudelaire en se voulant «parfait comédien» ${ }^{45}$; Villiers en assumant une conception très volontariste de l'identitét ${ }^{46}$. Rollinat semble pour sa part incapable d'une telle souplesse éthique; le caractère étroitement intensif de sa névrose le condamne à une forme d'éthos rigide et unidimensionnel - qui se traduit, sur le plan figural, dans la crispation comme catatonique, ou pantomimique en effet, de plusieurs de ses personnages poétiques (264, 265, 272, 273-276, 290, 291-292). Il proteste de sa bonne foi, répond aux accusations de cabotinage qu'on formule à son endroit, manifeste souvent un désir excessif de convaincre ${ }^{47}$, insiste sur l'ancienneté et l'authenticité de son mal, et ce, tout en multipliant, dans Les Névroses, les références parodiques, les exagérations macabres et les autres témoignages de l'esprit de jeu qui valent pour autant de signes d'appartenance très ostensibles à l'esthétique décadente. Sans doute la posture éthique que conditionne «ce Rollinat foncièrement sincère» ${ }^{48}$ entre-t-elle trop violemment en contradiction avec le peu de sérieux propre à l'esprit fin-de-siècle pour composer - au-delà de l'écrivain «à part» que Rollinat ambitionnait d'être et qu'il fut incontestablement ${ }^{49}$ - un personnage littéraire crédible, c'est-à-dire suffisamment cohérent pour susciter la croyance à son rôle, pour «fonctionner» dans l'économie de la croyance littéraire. Sans doute est-ce là une considération d'importance dans l'insuccès de l'auteur des Névroses et, incidemment, de sa tentative de retour/recours fin-de-siècle à la nature. On peut également y voir la raison pour laquelle, à ses pairs, Rollinat donnera l'impression d'être double, de n'être jamais tout à fait assimilable à leur monde éthique: «Il est des nôtres et n'est pas des nôtres» ${ }^{50}$, constate Émile Goudeau. Étrangeté qui obligera parfois ses critiques les mieux intentionnés à pousser loin la complaisance, à s'adonner à des acrobaties de lecture consistant par exemple à «raisonner» le texte au nom de la véracité présumée du discours biographique: ainsi, Gérard Peylet, après avoir cité quelques vers des Névroses d'une outrance toute décadente, se croit dans l'obligation d'ajouter: «Si nous ne connaissions pas la sincérité de Rollinat, nous pourrions croire à une parodie! $\gg^{51}$.

Par sa radicalité, la tentative d'hybridation de Rollinat a l'avantage critique de faire saillir un autre problème inhérent au décadentisme. Problème qui n'engage plus d'abord l'ethos et la posture d'énonciation du poète décadent, mais la logique de la sensation sur laquelle est fondée la possibilité même de l'expérience esthétique en régime décadent.

(44) Cfr. E.A. Poe, Contes, essais, poèmes, éd. Cl. Richard, Paris, Robert Laffont, 1989, «Bouquins», p. 1004.

(45) Cfr. Ch. Baudelaire, Euvres complètes, t. I cit., p. 1076.

(46) Conception qui s'exprime à travers les très nombreux énoncés de la fiction villiérienne enjoignant l'individu à choisir l'Idéal ou la Vérité susceptible d'en faire un dieu (cfr. L'Ėve future, Paris, Gallimard, 1993, «Folio Classique», p. 316).

(47) R. Miannay, Maurice Rollinat, poète et musicien du fantastique cit., p. 345.

(48) R. Bismuth, «Dans les brandes» de Maurice Rollinat et la bantise du genre bumain devant la nature, in L'Esprit de décadence. Colloque de Nantes (21-24 avril 1976), [sans mention d'éditeur], vol. I, Minard, 1980, «La Thèsothèque», p. 69.

(49) R. Miannay, Maurice Rollinat, poète et musicien du fantastique cit., p. 560.

(50) Cité par R. Miannay, ibid., p. 295.

(51) Les Evasions manquées ou les illusions de l'artifice dans la littérature «fin de siècle» cit., p. 171. 
Revenons, pour ce faire, à la névrotisation de la nature à laquelle nous avons fait allusion en première partie d'analyse. Cette opération esthétique est sans contredits l'aspect le plus original des Névroses. Quoiqu'elle implique une revalorisation insolite de thèmes naturels, qu'elle constitue un étonnant recours en forme de retour à la nature, elle s'inscrit dans le droit fil et même à la fine pointe du décadentisme. En décloisonnant la névrose et en faisant d'elle un attribut du monde naturel, Rollinat l'intensifie et, par là, se trouve obéir à la tendance lourde et caractéristique des temps modernes qu'est l'intensification ${ }^{52}$. On en conviendra, le frisson nerveux qu'il imprime à la nature répercute et illustre singulièrement bien, dans l'ordre de la représentation poétique, l'intensité que l'imaginaire moderne et plus nettement encore l'esprit fin-de-siècle célèbre en l'assimilant à une sorte de libido de la matière, à la puissance vitale d'un monde fantasmé comme naturellement électrique. Sa nature névrotique, en ce sens, est résolument d'époque, et il n'est pas exagéré d'affirmer, en soulignant dans l'expression une autre alliance de mots suggestive, qu'elle en est le plus pur produit. Elle porte l'hystérie moderne au dernier cri et au dernier degré de la logique de l'intensification.

On peut mieux apprécier l'intensité ou la modernité paradoxale de cette nature névrotique en la situant par rapport aux jeux de travestissement et de pervertissement du monde végétal auxquels s'adonne, au huitième chapitre d'À rebours, le protagoniste de Huysmans. On se souvient que, désireux d'aller au-delà de l'ambition générale et première du décadentisme, à savoir la dénaturalisation, le remplacement de la vie naturelle par la «vie factice» comme dirait Gautier ${ }^{53}$, Des Esseintes délaisse les fleurs artificielles au profit de fleurs véritables... donnant l'impression d'être fausses: «après les fleurs factices singeant les véritables fleurs, il voulait des fleurs naturelles imitant des fleurs fausses $\gg^{54}$. La nécessité où il est de raviver sa sensibilité toujours prompte à s'émousser, le porte ainsi à opérer un certain retour à la nature. Son plaisir est proportionnel à la subtilité de l'équivoque ainsi produite: «son but était atteint; aucune ne semblait réelle: l'étoffe, le papier, la porcelaine, le métal, paraissaient avoir été prêtés par l'homme à la nature pour lui permettre de créer des monstres» ${ }^{55}$. Tout en les précédant d'une année sur le plan chronologique, le poète des Névroses semble dépasser en intensité Huysmans et son personnage au jeu de la confusion du vrai et du faux: tout se passe comme si, chez lui, la nature n'avait plus même besoin d'être marquée du signe d'une quelconque exception pour satisfaire aux exigences de la sensibilité décadente; comme si elle y réussissait d'emblée, toujours déjà et globalement, dès lors qu'elle contenait sa propre altérité, son propre mouvement de différentiation à elle-même - le frisson - et, par suite, sa propre source de trouble excitation.

Évidemment, si tout devient objet à sensation, du fait de l'hystérie innée du poète et de la nature, rien ne l'est plus vraiment. C'est le critère même de l'expérience esthétique, la singularité qui fait la puissance d'excitation du «monstrueux» ${ }^{56}$, qui s'efface. C'est sous cet angle que la tentative poétique de Rollinat paraît confiner à la contradiction ou, pour être plus exact, par sa radicalité, trahir la contradiction

(52) Cfr. l'ouvrage récent de T. GARCiA, La Vie intense: une obsession moderne, Paris, Éditions Autrement, 2016.

(53) Théophile Gautier, in la notice de l'édition de 1868 des Fleurs du mal (Paris, Calmann-Lévy, 1868, p. 16).

(54) J.-K. Huysmans, À Rebours cit., p. 187.

(55) Ibid., p. 192.

(56) Sur la symbolique fondamentale du monstre, et incidemment de l'hybride et de l'«bubris», dans le décadentisme, cfr. E. STEAD, Le Monstre, le singe et le foetus. Tératogonie et décadence dans l'Europe fin-desiècle, Genève, Droz, 2004. 
interne au décadentisme: à savoir le fait que la valorisation décadente du faux se dédouble en une croyance à une nouvelle vérité, mais une vérité subjectivée et modalisée à l'extrême, et finalement invalidée, car corrélée à la sensation. Si cette contradiction recoupe une problématique plus vaste et bien connue en philosophie de l'art, inhérente au sensualisme, Catherine Coquio est l'un des rares critiques qui l'aient thématisée par référence au décadentisme:

Obsédée par le problème du faux et de l'authentique, l'écriture décadente est sur ce point profondément contradictoire: sa célébration du faux sacrifie, en creux, à la croyance du vraie, vidée de tout contenu. [...] Le vrai s'est réfugié dans le corps, et son seul mode d'évaluation est la sensation, dont le décadent, comme l'écrit Jean Lorrain, devient le «forçat». Mallarmé écrit à Lefébure à propos d'Elën de Villiers: «Vous ressentirez une sensation à chacun des mots, comme en lisant Baudelaire». [...] Or, la sensation est le noyau trouble qui contient virtuellement tous les brouillages du vrai et du faux, puisqu'on peut la susciter à volonté, comme Des Esseintes en fait l'expérience méthodique ${ }^{57}$.

Au contraire du symbolisme qui, quelques années plus tard, avec son subjectivisme radical et, sur le plan institutionnel, son foisonnement de revendications anomiques, sera le premier mouvement à professer un relativisme extrême en matière d'art, le décadentisme a encore besoin, en principe, de certaines assises ontologiques: il croit au faux, à la vérité du faux. L'univers esthétique, au sens fort de l'adjectif, qu'il détermine en se détournant des critères rationnels du classicisme érige le faux au rang d'une vérité qui renvoie potentiellement à tout - ce qui peut être senti. Chez Rollinat, cette vérité sensitive est non seulement attestable et décrétable «à volonté», selon les lubies du poète: elle s'indique partout, puisque tout, la nature aussi bien que le poète, est transi par la tension et le frisson esthétiques. Du coup, l'expérience esthétique s'y banalise; elle perd la rareté qui en fait par ailleurs la valeur: elle n'est plus le produit d'un patient travail d'élaboration, comme c'est le cas chez Des Esseintes, pas plus qu'elle ne se réserve aux soudains coups de folie où, l'instant d'un moment, l'artiste baudelairien croit «trouver» l'«infini de la jouissance» ${ }^{58}$. Cette banalisation est aussi, fatalement, celle de l'expérience esthétique de la lecture, et on comprend que le volumineux recueil des Névroses, avec ses innombrables scènes d'horreur indéfinie et comme indéfiniment répétable, ait davantage irrité que stimulé les nerfs de beaucoup de ses lecteurs.

PATRICK THÉRIAULT

University of Toronto

(57) C. Coquio, La «Baudelairité» décadente: un modèle spectral cit., pp. 97-98.

(58) Ch. Baudelaire, Euvres complètes, t. I cit., p. 287. 